\title{
A rapid, simple method for the genetic discrimination of intact Arabidopsis thaliana mutant seeds using metabolic profiling by direct analysis in real-time mass spectrometry
}

Suk Weon $\mathrm{Kim}^{1+}$, Hye Jin Kim ${ }^{3+}$, Jong Hyun Kim², Yong Kook Kwon ${ }^{1}$, Myung Suk Ahn², Young Pyo Jang ${ }^{3^{*}}$ and Jang R Liu $2^{2^{*}}$

\begin{abstract}
Background: Efficient high throughput screening systems of useful mutants are prerequisite for study of plant functional genomics and lots of application fields. Advance in such screening tools, thanks to the development of analytic instruments. Direct analysis in real-time (DART)-mass spectrometry (MS) by ionization of complex materials at atmospheric pressure is a rapid, simple, high-resolution analytical technique. Here we describe a rapid, simple method for the genetic discrimination of intact Arabidopsis thaliana mutant seeds using metabolic profiling by DART-MS.

Results: To determine whether this DART-MS combined by multivariate analysis can perform genetic discrimination based on global metabolic profiling, intact Arabidopsis thaliana mutant seeds were subjected to DART-MS without any sample preparation. Partial least squares-discriminant analysis (PLS-DA) of DART-MS spectral data from intact seeds classified 14 different lines of seeds into two distinct groups: Columbia (Col-0) and Landsberg erecta (Ler) ecotype backgrounds. A hierarchical dendrogram based on partial least squares-discriminant analysis (PLS-DA) subdivided the Col-0 ecotype into two groups: mutant lines harboring defects in the phenylpropanoid biosynthetic pathway and mutants without these defects. These results indicated that metabolic profiling with DART-MS could discriminate intact Arabidopsis seeds at least ecotype level and metabolic pathway level within same ecotype.
\end{abstract}

Conclusion: The described DART-MS combined by multivariate analysis allows for rapid screening and metabolic characterization of lots of Arabidopsis mutant seeds without complex metabolic preparation steps. Moreover, potential novel metabolic markers can be detected and used to clarify the genetic relationship between Arabidopsis cultivars. Furthermore this technique can be applied to predict the novel gene function of metabolic mutants regardless of morphological phenotypes.

Keywords: Arabidopsis thaliana, Direct analysis in real-time mass spectrometry (DART-MS), partial least squaresdiscriminant analysis (PLS-DA), seed

\footnotetext{
* Correspondence: ypj0924@gmail.com; jrliu@kribb.re.kr

† Contributed equally

${ }^{2}$ Plant Systems Engineering Research Center, Korea Research Institute of

Bioscience and Biotechnology (KRIBB), 125 Gwahak-ro, Yuseong-gu, Daejeon,

305-806, Korea

${ }^{3}$ Division of Pharmacognosy, Kyung Hee University, 1 Hoegi dong

Dongdaemun-Gu, Seoul, 130-701, Korea

Full list of author information is available at the end of the article
} 


\section{Background}

Functional genomics of higher plants is conducted primarily using a phenotype-based approach. A knockout or over-expressed gene is assumed to produce an overt phenotype in a model plant. However, in practice a large proportion of mutants show no visible morphological phenotype or the phenotype results from a secondary or pleiotropic change, which hinders identification of the gene function. To achieve the practical goal of functional genomics, a more robust characterization system is required to identify mutants.

Global metabolic profiling coupled with statistical analysis is often used for diverse plant biotechnology applications including rapid discrimination between plant species [1-3], differentiation of cultivars or ecotypes [4-6], identification of genetically modified plants [7-13], and metabolic evaluation of commercial food stocks or medicinal herbs [14-18]. These techniques have also been successfully applied to plant functional genomics [19-22]. Recently, plant metabolomics combined with transcriptomics has been used to characterize systemic physiological stress responses [23-26].

Metabolic profiling, which can reveal the metabolic phenotypes of mutants, is most often carried out using gas chromatography (GC)/MS, liquid chromatography (LC)/MS, and proton nuclear magnetic resonance $(1 \mathrm{H}$ NMR) [27]. For example, Messerli et al. [11] reported that metabolite fingerprinting with GC-MS differentiated Arabidopsis mutants defective in starch metabolism from other mutants, indicating that non-targeted metabolic profiling of mutants provides clues about the mutated gene(s). However, it is difficult to achieve high throughput with these instruments primarily due to complicated sample preparation, large sample requirements, and time-consuming operation. Recently, DARTMS has been used for a non-invasive, high-throughput metabolic profiling of samples from various organisms. This new MS technique does not require sample preparation or vacuum ionization, making it an extremely versatile high-throughput system [28-30].

DART ion source is a recently developed ambient ion source which can ionize various organic molecules in diverse samples directly from the surface. In open air conditions, helium, as a carrier gas, produces protonated water clusters from atmospheric water molecules then transfer the proton into molecules in the samples [28]. DART ion source is especially powerful when it is combined with high resolution mass analyzer as it gives exact molecular weight of ionized compounds from the samples and provides matching molecular formula thereof. DARTMS has been adopted in various qualitative analysis of organic molecules including pharmaceuticals, metabolites, synthetic organic molecules and phytochemicals [31-33].
For the quantification purpose, well-known anti-oxidative natural product, curcumin was successfully quantitated from raw material directly [34]. Furthermore, analytical reproducibility of DART-MS was also confirmed using caffeine-d3 with TLC analysis and [35] and olive oil ion analysis [36]. Since labor-taking sample preparation steps can be omitted in DART-MS analysis, high throughput fingerprinting study of natural resources is possible and this feature is one of the most advantageous characteristic of DART ion source in metabolomics approach. And DART ion source ionize moderately polar to highly nonpolar compounds [35]. Therefore phenylpropanoids are easily ionized and well-suited to detection.

In this study we attempted to establish high-throughput discrimination with DART-MS to detect intact mutant seeds. To determine whether metabolic profiling with DART-MS can discriminate seeds based on ecotype and altered metabolism, we used 14 different lines of Arabidopsis mutant seeds in two different ecotype backgrounds, including previously identified knockout mutants of the phenylpropanoid biosynthetic pathway.

\section{Results and Discussion}

\section{DART-MS spectra from Arabidopsis seeds}

Representative DART-MS spectra from intact seeds of two Arabidopsis ecotypes, Col-0 and Ler are shown in Figure 1. More than 319 peaks were detected from intact seed by DART-MS analysis (Additional file 1). Spectral differences between the two ecotypes were significant. Intact seeds of Col-0 ecotypes produced more prominent peaks compared to those of Ler ecotypes. These results implied that there were quantitative and qualitative differences in metabolite patterns between two Arabidopsis ecotypes. Interestingly, intact seeds of Col-0 and Ler ecotypes could be successfully discriminated using DART-MS spectral data combined by PLSDA, even though there is no apparent morphological differences (size, color, or shape) in seed morphology with naked eye (Figure 2). However, principal component analysis (PCA) could not fully differentiate these groups (Additional file 2). In cross-validation, each case was classified by the functions derived from all other cases; $100 \%$ of original grouped cases and $100 \%$ of cross-validated grouped cases were correctly classified (Table 1). These results indicated that metabolic profiling with DART-MS spectral data could discriminate two Arabidopsis ecotypes even though there were no visible, distinctive characteristics in the seed morphology.

\section{Genetic discrimination of two ecotype backgrounds from DART-MS spectral data of intact seeds}

Identification of gene function is limited when based on mutant morphological phenotype, which is often silent 

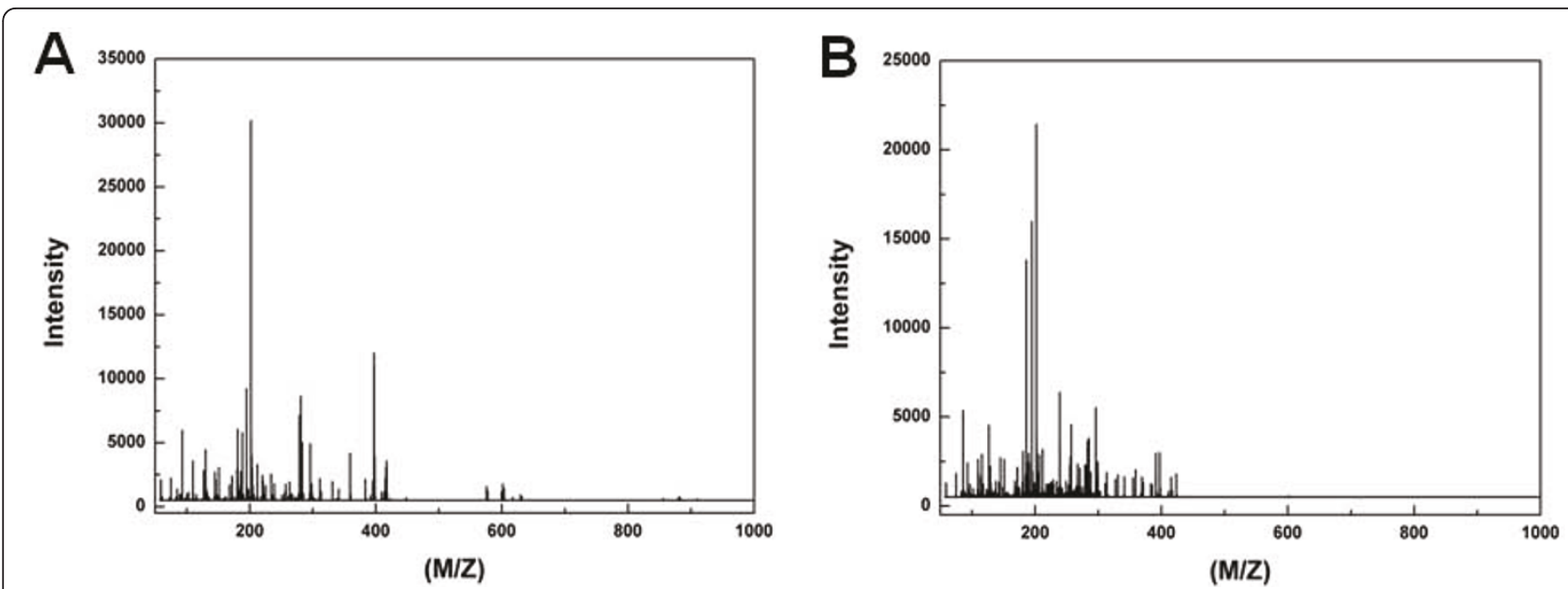

Figure 1 Representative direct analysis in real-time mass spectrometry (DART-MS) spectra from intact seeds of Col-0 (A) and Ler (B) ecotypes.

or the result of a secondary or pleiotropic changes. To overcome this limitation, non-targeted metabolic profiling of the mutant may provide clues about the mutated gene(s). To investigate the discrimination possibility between genetic defective mutants from same background, we applied three Aabidopsis mutants (co, ft and tt2) from Landsberg background and nine mutants (chs, f3h, f3'h, dfr, ldox, ban, pap1-D, phyB, and ugt78d2) from Col-0 in this study.

$c o$ and $f t$ are representative Aabidopsis mutants showing late flowering phenotype that is null mutants of CONSTANS (CO) and FLOWERING LOCUS T (FLC), respectively [37]. $t t 2$ is the null mutant of

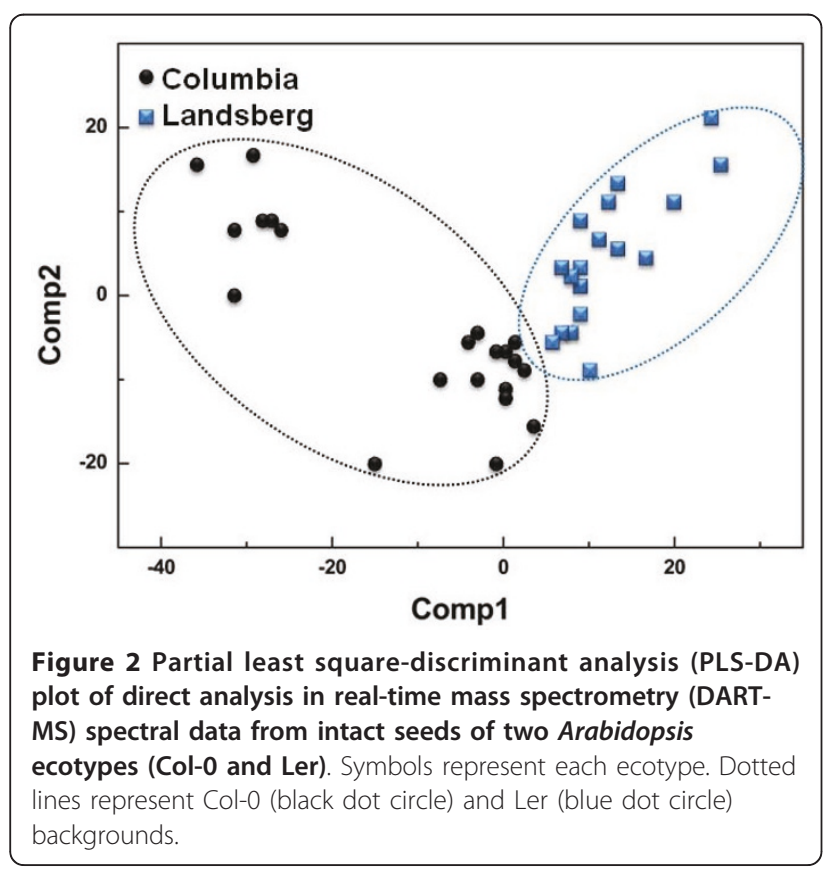

TRANSPARENT TESTA 2 (TT2), that is one of R2R3 MYB domain transcription factor and acts as a key determinant in the proanthocyanidin accumulation of developing seed. It is composed of ternary complex with TT8 and TTG1 for correct expression of BANYULS (BAN) in seed endothelium [38-40]. Therefore the seed color of $t t 2$ mutant is yellow due to the defectiveness of condensed tannin accumulation in the seed endothelium.

Nnine Arabidopsis mutants (phyB, pap1-D, chs, f3h, $f 3^{\prime} h, d f r, l d o x, b a n$, and $\left.u g t 78 d 2\right)$ are belonging to Col-0 background. $p h y B$ and pap1-D are a null mutant of phytochrome B (PHYB), primary red/far-red photoreceptor in light signaling transduction pathway and an activated mutant of PRODUCTION OF ANTHOCYANIN PIGMENT 1 (PAP1) act as an activator of phenylpropanoid biosynthesis, respectively $[41,42]$. The rest of mutants belonging to Col-0 background are seven T-DNA inserted knock-out mutants (chs, f3h, f3'h, dfr, ldox, ban, and ugt78d2) involved in anthocyanin biosynthesis pathway. The gene expression levels of these mutants (chs, $f 3 h, f 3^{\prime} h, d f r$ and $\left.l d o x\right)$ were analyzed by RT-PCR using

Table 1 Cross validation between two Arabidopsis ecotypes from direct analysis in real-time mass spectrometry (DART-MS) spectral data of intact seeds

\begin{tabular}{llccc}
\hline Intact seed & & \multicolumn{2}{c}{ Prediction } & \multirow{2}{*}{ Total } \\
\cline { 2 - 4 } & & Col-0 & Ler & \\
\hline Cross-validated count & Col-0 & 21 & 0 & 21 \\
\hline Ler & 0 & 21 & 21 \\
\hline Total & & 21 & 21 & 42 \\
\hline
\end{tabular}

The discriminant functions were the first five discriminant components from PLS-DA analyses ( 5 data). In cross-validation, each case was classified by the functions derived from all other cases; $100 \%$ of original grouped cases and $100 \%$ of cross validated grouped cases were correctly classified. 
primer pairs for pull length ORF (Figure 3). This result indicated that all of the mutants were completely defective mutants. But DFR was slightly expressed in $d f r$ mutant line, probably resulting from T-DNA insertion on the 3' UTR region of DFR gene. No expression of BAN was observed in both WT and ban mutant seedlings as previous reported by Lee et al. [43]. Three mutants could easily be discriminated by the seed color itself. The color of seed coat from $t t 2$ and chs mutants was yellow, whereas that of pap1-D mutant was darker than wild type. Although rest of mutants used in this study has slightly difference in seed color, it could not easily be discriminated without direct comparison with wild type. Furthermore, determination of genetic background from $t t 2$, chs and pap1-D mutants was impossible by naked eye, even though they had an obvious seed color.

PLS-DA of DART-MS spectral data from intact seeds divided the 14 lines of seeds into two distinct groups: Col-0 and Ler ecotype backgrounds (Figure 4). In crossvalidation, each case was classified by the functions derived from all other cases; $100 \%$ of original grouped cases were correctly identified, whereas $80.6 \%$ of crossvalidated grouped cases were correctly classified (Table 2). In particular, the mutant seed lines chs, co, and $f t$ were perfectly predicted, whereas $d f r$ was incorrectly classified. What $d f r$ shown comparatively low level of accuracy in cross-validation thought to be partial production of metabolic components caused by slightly expressed DFR in the mutant. In contrast, few prediction errors were made at the ecotype level by analysis of intact seeds. All mutant lines (chs, f3h, f3'h, dfr, ldox, ban, pap1-D, phyB, and ugt78d2) and wild type seeds belonging to the Col-0 ecotype were correctly predicted within the Col-0 ecotype. Likewise, mutant lines ( $c o$ and $f t$ ) and wild type Ler seeds were correctly predicted within the Ler ecotype. However, prediction of the $t t 2$ line was $90.5 \%$ accurate. These results indicated that DART-MS spectrometry combined with multivariate analysis of intact seeds was able to discriminate the lines of seeds at least the ecotype level.

In the present study, we described a robust method for high throughput profiling with DART-MS. The functional identification of genes of mutant

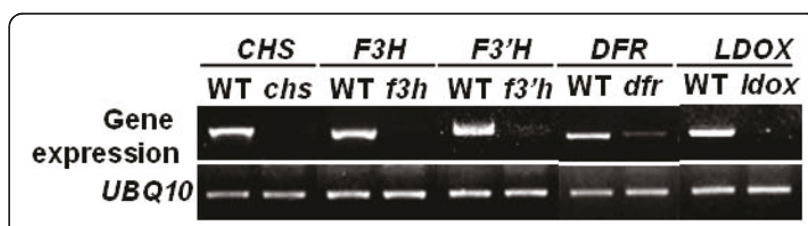

Figure 3 RNA expression level in each T-DNA inserted mutant lines. Total RNA were extracted from seven-day old grown seedlings. Gene expression level of each mutant line was analyzed with primers for full length ORF by RT-PCR.

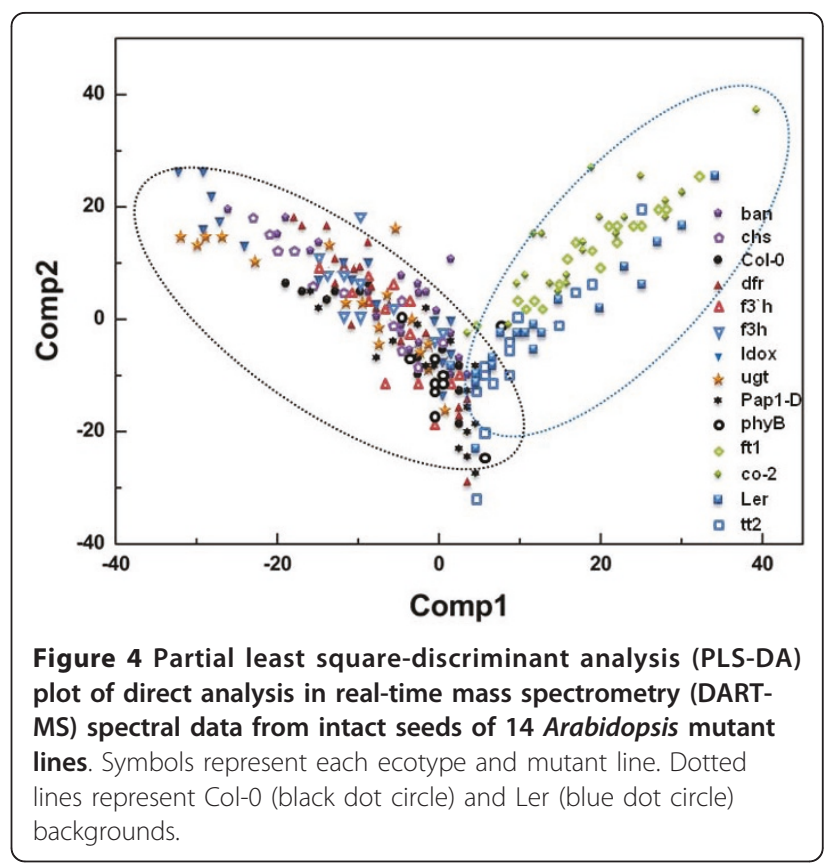

Arabidopsis is currently based on morphological phenotype. However, up to $85 \%$ of the mutants exhibit no overt phenotype [21]. Metabolic alterations may be present in a large portion of these mutants; however, current methods for metabolic profiling are complicated and time-consuming, which precludes highthroughput screening of mutants. In the present study, a robust screening of mutants with altered metabolism was devised with DART-MS using intact Arabidopsis mutant seeds. This approach is not limited to screening mutants lacking genes expressed in the seed coat, but may be extended to identification of genes expressed in other seed parts. DART-MS has been used to identify plant compounds [29,30], but this rapid, simple instrumentation has not yet been utilized for high-throughput screening of mutants. Considering the overall PLS-DA results, intact Arabidopsis seeds could allow genetic discrimination of ecotypes and sorting of specific mutants harboring defects in the phenylpropanoid biosynthetic pathway (Figures 2 and 4) and flowering time genes.

\section{Hierarchical clustering of Arabidopsis seeds based on} multivariate analysis of metabolic profiling

A hierarchical dendrogram based on PLS-DA of DARTMS spectral data from intact seeds showed that 13 lines (excluding the tt2 mutant line) were divided into two major branches by ecotype (Figure 5). Interestingly, the hierarchical dendrogram from intact seed subdivided the Col-0 ecotypes into two subgroups: seven mutant lines lacking a gene involved in the phenylpropanoid biosynthetic pathway except the ugt78d2 clustered together, 
Table 2 Cross validation of Arabidopsis ecotypes from direct analysis in real-time mass spectrometry (DART-MS) spectral data of intact seed

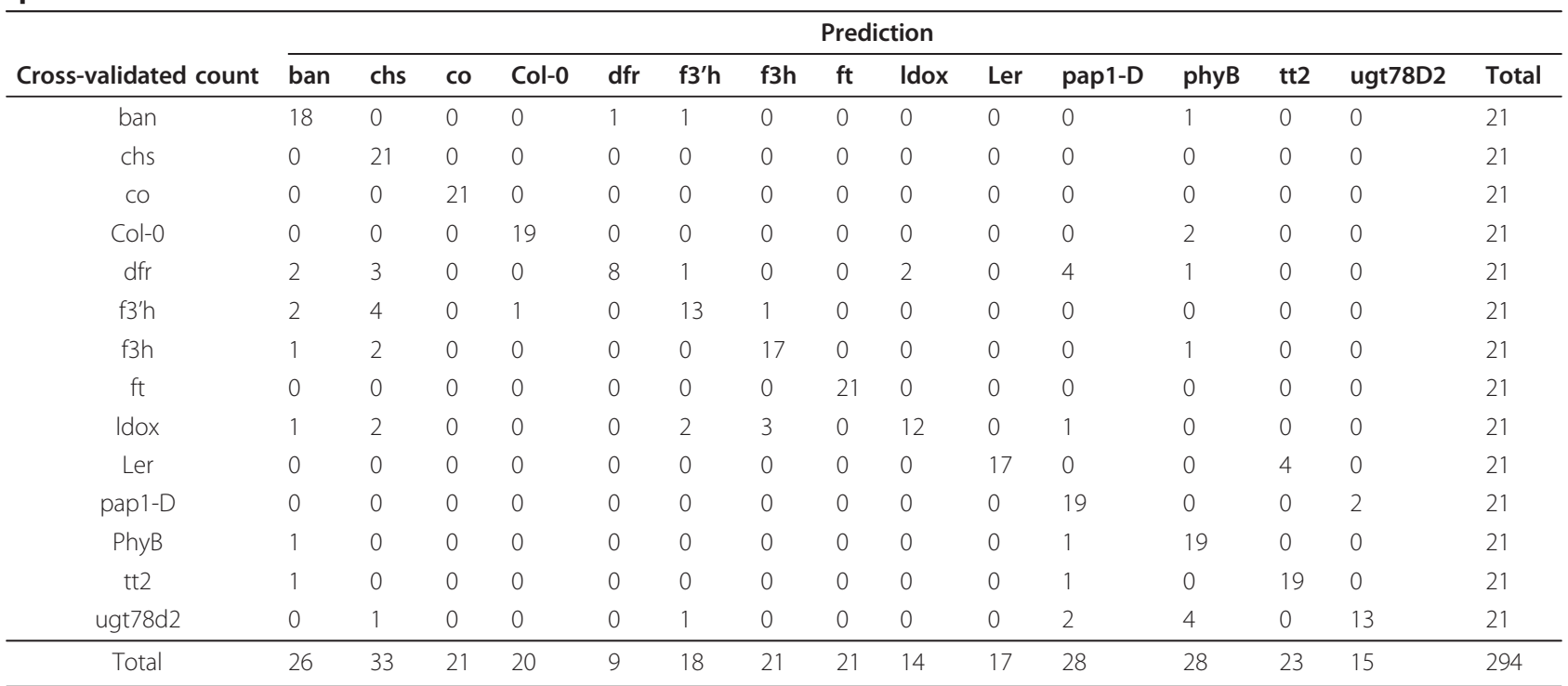

The discriminant functions were determined using the first five discriminant components determined by PLS-DA analyses (5 data). In cross-validation, each case is classified by the functions derived from all other cases; $100 \%$ of original grouped cases, while $80.61 \%$ of cross validated grouped cases were correctly classified.

separate from the other three lines ( $p h y B$, pap1-D, and Col-0) (Figure 5).

Anthocyanins are water-soluble vacuolar pigments and belong to a parent class of flavonoids synthesized via the phenylpropanoid pathway. Anthocyanins are found in all tissues of higher plants, and especially proanthocyanidins in the seed coat. UGT78D2 that catalyzes the glucosylation of both flavonols and anthocyanidins which converted to anthocyanins, is highly expressed in

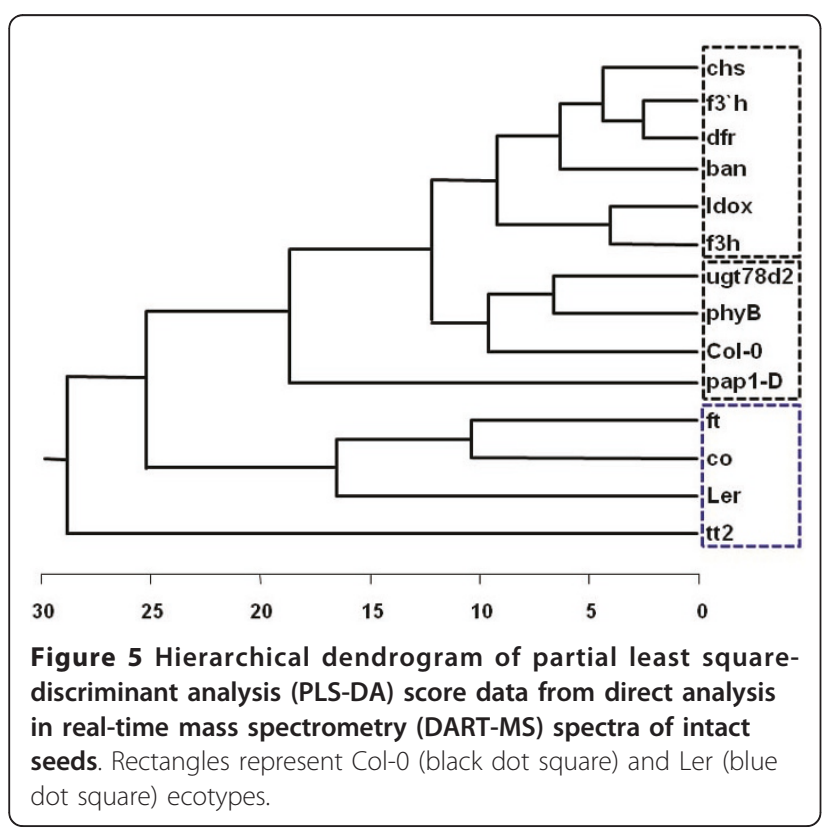

anthocyanin-accumulating seedlings, but repressed in condensed tannin-accumulating seed coats [43]. It seems that metabolic flux for the metabolic end-products in mature seed coats is not affected even if UGT78D2 is abolished in Arabidopsis seedlings or seeds. It indicated that there might be no differences in metabolic components between WT and ugt78d2 mutant seed coats. BAN encodes a core enzyme, anthocyanin reductase of flavonoid biosynthesis. It is convert anthocyanindins to flavan-3-ol, which condensed to colorless proanthocyanins [44]. They are placed only in the seed coat, and confer a brown color to mature seed after oxidation. BAN, also act as a negative regulator of flavonoid biosynthesis during early embryogenesis, and highly expressed in the tannin-accumulating mature seed in Arabidopsis [43,45-47]. Therefore, accumulation of proanthocyanins probably is inhibited in the developing seed coats of ban mutants. From this point of view, it is reasonable that $u g t 78 d 2$ grouped with WT, and ban clustered with chs, $d f r$ and $f 3^{\prime} h$, respectively. Therefore, we inferred that the combination of multivariate analysis and DART-MS, might reflect gene functional relationship on flavonoid biosynthetic pathway such as ugt78d2 and ban clustered with other associated mutants reasonably.

A hierarchical dendrogram based on PLS-DA of DART-MS spectral data from intact seeds from Ler and three mutant lines ( $c o, f t$ and $t t 2$ ), they were separated into other major branches from Col-0 ecotypes except for the $t t 2$ mutant line (Figure 5). TT2 functions as a regulator in proanthocyanidin accumulation in 
developing seed only when TTG1 is expressed. The ternary complex of TT2, TT8 and TTG1 positively regulate BAN expression in whole seed coats by directly regulating $B A N$ promoter activity in plants $[39,40,48]$. No detection of $B A N$ transcript in $t t 2$, $t t 8$ and $t$ tg 1 confer homogeneously yellow hue on their seed coats $[39,47]$. Therefore, we expected that $t t 2$ mutant was clustered with other $t t$ mutants in the fravonoid biosynthetic pathway, especially ban mutant, regardless of background properties. However, $t t 2$ was placed near to Ler branch from the hierarchical dendrogram even though $t t 2$ was not included into Ler branch (Figure 5). These result implied that overall metabolic differences between Arabidopsis ecotypes was greater than that of a specific gene, for example metabolic change in tannin accumulation of tt 2 mutant. Therefore we suggested that there were common metabolic compounds that mainly affected for the ecotype discrimination of Arabidopsis. Considering the overall hierarchical clustering analysis (HCA) results, we concluded that DART-MS spectrometry combined with multivariate analysis of intact seed could not only discriminate Arabidopsis seeds at the ecotype level, but could also cluster metabolic genes related to same metabolic pathway. Therefore, we suggest that DART-MS spectrometry may be useful as a tool for rapid discrimination of ecotypes and metabolic mutants of Arabidopsis.

\section{Assignments of chemical compounds for ecotype discrimination}

Mass spectrometry analysis is one of the most powerful analytical methods available for exact structural identification of organic compounds. In this study, more than 319 peaks were detected from intact seed by DART-MS analysis (Figure 1$)$. These peaks $(\mathrm{m} / \mathrm{z}$ ) have not fully assigned as chemical compound yet, because of lack of Arabidopsis chemical DB. We selected the top 10 most significant metabolites for discrimination between Col-0 and Ler ecotypes from the MS spectral data of intact seeds by logistic regression (Figure 6).

In general, DART ion source commonly produces a mass spectrum consisting of the $[\mathrm{M}+\mathrm{H}]^{+}$molecular cation by proton transfer mechanism. But molecular ion peak of $\mathrm{M}+$ also found commonly by penning reaction. Thus, the ion peak of selected compounds in this study was assigned as molecular ion by penning reaction. However, DART-MS cannot discriminate the same chemical formula compounds similar to all other mass spectrometers. Therefore, with the works of intensive in silico informatics on previous phytochemical studies on Arabidopsis thaliana only gives the information on exact molecular weights for the compounds in samples. Assignment of the 10 metabolites was performed by direct comparison with the online chemical database
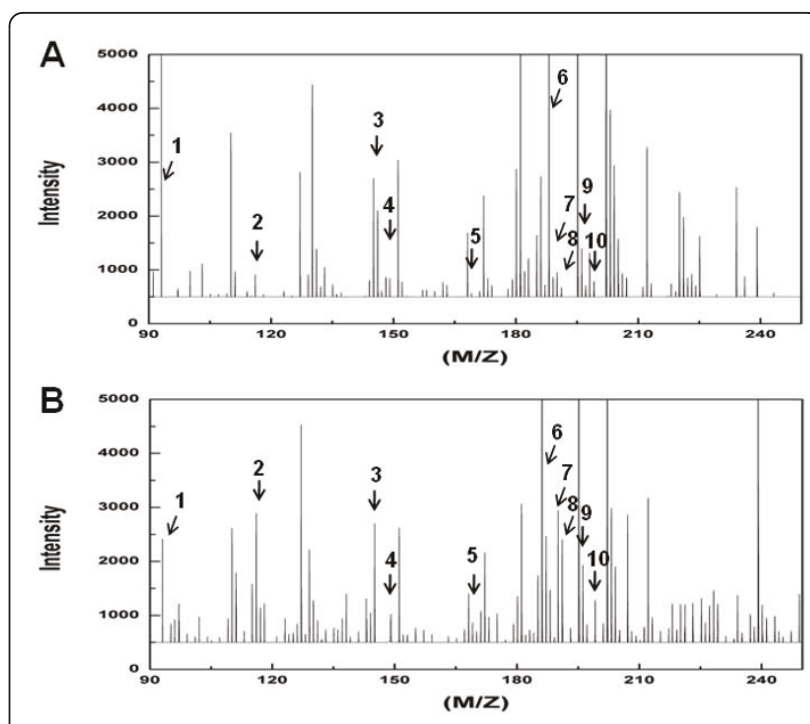

Figure 6 Enlarged view of direct analysis in real-time mass spectrometry (DART-MS) spectra from intact seeds of Col-0 (A) and Ler (B) ecotypes. Arrows and numbers represent 10 major compounds for metabolic discrimination between the ecotypes.

Plant Metabolic Network (http://www.plantcyc.org) (Table 3).

The intensity of the molecular ion peak of glycerol $(\mathrm{MW}=92.115)$ was 2.5 times higher on the seed surface of the Col-0 ecotype than that of Ler. The intensity of dimethyl fumarate $(\mathrm{MW}=145.106)$ was 3.5 times higher in seed surface of the Col-0 ecotype than that of Ler. Whereas molecular ions of L-glutamic acid, pyridoxal and 5-hydroxyconiferyl aldehyde was more abundant in Ler than in the Col-0 ecotype. Ward et al. [4] reported that nine Arabidopsis ecotypes could be discriminated based on glucose and fumaric acid content by ${ }^{1} \mathrm{H}$ NMR spectroscopy. Direct comparison of

Table 3 Ten major metabolites used for ecotype discrimination from intact seed of Arabidopsis ecotypes

\begin{tabular}{lccc}
\hline No & Compound & $\mathrm{m} / \mathbf{z}^{\mathbf{a}}$ & $\begin{array}{c}\text { Ratio(Col-0/ } \\
\text { Ler) }\end{array}$ \\
\hline 1 & Glycerol & 92.115 & 2.465 \\
\hline 2 & L-delta1-Pyrroline-5-carboxylate & 114.104 & 0.466 \\
\hline 3 & Dimethyl fumarate & 145.106 & 3.526 \\
\hline 4 & L-Glutamic acid & 148.107 & 0.665 \\
\hline 5 & Pyridoxal & 168.158 & 0.651 \\
\hline 7 & NA & 185.155 & 0.236 \\
\hline 8 & N-Methoxyindole 3-carboxylic acid methyl & 190.191 & 0.351 \\
\hline 9 & ester & 186.170 & 0.378 \\
\hline 10 & 5-Hydroxyconiferyl aldehyde & 195.163 & 0.608 \\
\hline
\end{tabular}

${ }^{a}$ mass-to-charge ratio. ${ }^{b}$ Not assigned for pure compound. 
key metabolites between the report of Ward et al. [4] and this study were not suitable because of difference in organic solvent and plant materials for metabolite extraction steps. In the present study, we conducted direct MS analysis of seed surface without any organic solvent extraction steps. However dimethyl fumarate was the one of key metabolite in seed coat of Col-0 ecotype. We have not fully understood the biochemical and metabolic pathway of fumaric acid and dimethyl fumarate, especially in seed coat yet. If fumaric acid could be actively modified by methyl group in seed coat, the report of Ward et al. [4] and our study showed that fumaric acid derivatives were key metabolites for ecotype discrimination in Arabidopsis. In addition, we also found that glycerol had a key role in ecotype discrimination of Arabidopsis. Therefore, the report of this study could be applied for the study of glycerol biosynthesis pathway in Arabidopsis ecotypes.

Although other MS with higher analytical resolution such as FT-ICR MS can be used for this study, they are suitable for the analysis of unknown species owing to its ultra high mass resolution and accurate mass capacity [49]. AccuTOF, the analyzer used in this study, is an orthogonal acceleration time-of-flight mass spectrometer (oa-TOF-MS) incorporating a single stage reflectron. The resolving power of this analyzer is excess of mass 6,000 (FWHM definition) [50]. Taking into account the relatively high resolution, and fast scan speed with a wide dynamic range, AccuTOF-MS is a powerful tool for high-throughput profiling or chemical fingerprinting of intact seed samples.

\section{Conclusions}

In this study, we demonstrate that DART-MS combined by multivariate analysis allows for rapid screening and metabolic characterization of lots of Arabidopsis mutant seeds without complex metabolic preparation steps. Our results represent that mutant lines including wild types were classified to two distinct groups, Col-0 and Ler ecotype backgrounds on PLS-DA from DART-MS spectral data of 14 lines of intact Arabidopsis seed. Furthermore, mutants which are Col-0 background were subdivided into two groups in the hierarchical dendrogram based on PLS-DA, in which one group of defective mutants is related to the phenylpropanoid biosynthetic pathway. These results demonstrate that DART-MS combined by multivariate analysis can discriminate mutants based on quantitative and qualitative differences affecting global metabolic profiles. Considering these results we infer that metabolic profiling with DART-MS could discriminate intact Arabidopsis seeds at least ecotype level or metabolic pathway level within same ecotype. Screening mutants in the form of seeds saves the labor time required to grow plants. Thus, we suggest that DART-MS spectrometry combined by multivariate analysis is a useful tool for only rapid screening of metabolic mutants, but also discrimination of ecotypes of Arabidopsis. Furthermore plant functional genomics can be carried out based on metabolic profiling of intact Arabidopsis mutant seeds by DART-MS in a high throughput manner.

\section{Materials and methods Plant materials}

Arabidopsis plants were grown in a growth room at 20 to $24^{\circ} \mathrm{C}$ under a long day condition $(16 / 8 \mathrm{hr}$ photoperiod at $80 \mathrm{umol} \mathrm{m}^{-2} \mathrm{~s}^{-1}$ ) with white light in the Arasystem (LEHLE SEEDS. USA). All of the seeds were harvested and desiccated for 2 weeks at $30^{\circ} \mathrm{C}$ chamber for this study. Col-0 and Ler ecotypes were used as wild type of Arabidopsis thaliana. We applied three mutants as the mutant of Ler ecotype background; co (co-2, cs175), $\mathrm{ft}$ ( $\mathrm{ft}-1, \mathrm{cs} 56)$, and $t \mathrm{t} 2$ (tt2-1, cs83), and nine mutants as that of Col-0 ecotype background; phyB (phyB-9, cs6217) pap1-D (cs3884), chs (salk_064816), f3h (salk_113904), f3'h (salk_124157), dfr (cs115210), ldox (salk_073183), ban (salk_040250) and ugt78d2 (ugt78d21, salk_049338, [41]). These mutants except T-DNA insertion lines (SALK lines) were gained from the Arabidopsis Biological Resource Stock Center [51]. T-DNA inserted mutants (chs, f3h, f3'h, dfr, ldox, ban and ugt78d2), also derived from ABRC were kindly provided by Dr. Giltsu Choi (KAIST, Daejeon, Korea), in which T-DNA inserted position were previously confirmed on the genome of each mutants by PCR method using each genes primer and LBa1 primer for the T-DNA vector, pROK2 (http://signal.salk.edu/), and then selected homozygous T-DNA inserted mutants. To confirm the RNA expression of T-DNA inserted lines, we extracted total RNA from the seven-day-old white-light grown seedling with RNeasy Miniprep Kit (Qiagen, Germany), and reverse-transcribed with M-MLV reverse transcriptase and Oligo $(\mathrm{dT})_{15}$, according to the manufacturer's protocol (Enzynomics, KOREA). They were equalized by ubiquitin (UBQ10; AT4G05320, 5'-AGTCCACACTTCACTTGGTC-3', 5'-TTAGAAACCACC ACCGA-3'). Primers for expression analysis included: CHS (At5g13930, 5'-TCCCCCGGGC ATGGTGATGGCTGG TGCTTCTT-3', 5'-CCGCTCGAGGAGAGGAACGCT GTGCAAGAC G-3'), F3H (At3g51240, 5'-TCCCCCG GGCATGGCTCCAGGAACTTTGACTG-3', 5'-CGGGA TCCGAAGCGAAGATTTGGTCGACAG GC-3'), F3'H (At5g07990, 5' - TCCCCCGGGCATGGCAACTCTATT TCTCACAATC-3', 5'-CCGCTCGAGACCCGACCCG AGTCCATAAACG-3'), DFR (At5g42800, 5'-TCCCCCG GGCATGGTTAGTCAGAAAGAGACC-3', 5'CGGGAT CCGAGGCACACATCTGTTGTGCTAGC-3'), LDOX (At4g22880, 5'-TCCCCCGGGCATGGTTGCGGTTG 
AAAGAGTTGA-3', 5'-CGGGATCCGAATCATTTT TCTCGGATACCAATTC-3'), BAN (AT1G61720, 5'TCCCCCGGGCATGGACCAGCTCTTACACACAC-3', 5'-CCGCTCGAGT TTAGCTTTGA TCAATCCTTT TGA-3')

\section{DART-MS}

A Jeol DART-MS instrument (Tokyo, Japan) was used, which comprised a DART ion source and a JMST100TD (AccuTOF) atmospheric pressure ionization time-of-flight mass spectrometer. For positive ion detection, the atmospheric pressure interface potentials were set to the following values: orifice $1=10 \mathrm{~V}$, ring lens and orifice $2=5 \mathrm{~V}$. The ion guide potential and detector voltage were set to $500 \mathrm{~V}$ and $2400 \mathrm{~V}$, respectively. DART electrode potentials were set to needle electrode $=3000 \mathrm{~V}$, electrode $1=100 \mathrm{~V}$, electrode $2=100 \mathrm{~V}$. Gas temperature was set to $250^{\circ} \mathrm{C}$, and the helium gas flow rate was $3 \mathrm{~L} / \mathrm{min}$. Each seed was positioned midway between the DART source and mass spectrometer for measurement. Three measurements for each seed were averaged, and three different seeds of each wild type and mutant line were used as replicates.

\section{Data processing and multivariate statistical analysis}

To minimize the influence of sample size, DART-MS spectral data were normalized to total ion count percent. Small noise peaks with low ion intensity value $(<100)$ were removed from the original spectral data. Multivariate statistical analysis was performed using mean-centered and auto scaled data. These preprocessed metabolomic datasets were imported into $\mathrm{R}$ programs for PCA, PLS-DA and HCA. PCA, an unsupervised clustering method, was performed to statistically analyze comprehensive information contained in a data set [52]. Also PLS-DA, a representative supervised data mining algorithm, could give more precise group separation. To create the PLS-DA model, the entire data set is divided into two parts: a training set that was used to build a model, and a test set that was not used in the classification model, but was used to verify the model's predictive ability. To estimate the predictive power and significance of a latent variable of a model, cross-validation was used. Permutation testing also evaluates the statistical significance of the estimated predictive power of a model. After predictive validation by means of cross-validation and response permutation testing, external validation, a more demanding and rigorous mechanism for testing predictive performance consisted of computing predictions for an independent set of test observations (test set). Also, HCA was performed to statistically analyze comprehensive relationship contained in PLS-DA score data from each sample.
To identify the significant metabolites for ecotype discrimination from MS spectral data, P-values for all metabolites were calculated by logistic regression, and 10 metabolites with high P-values were selected. Assignment of 10 metabolites was conducted by comparison with the database of the Plant Metabolic Network (http://www.plantcyc.org).

\section{Additional material}

Additional file 1: Raw data of DART-MS spectra from intact 14 Arabidopsis mutant seeds. MS spectral data was consisted of three replicates from each seed line.

Additional file 2: PCA score plot of DART-MS spectra from two Arabidopsis ecotypes (Col- 0 and Landsberg)

\section{List of abbreviations}

ASCII: American standard code for information interchange; Col-0: Colombia; DART-MS: Direct analysis in real-time mass spectrometry; HCA: hierarchical clustering analysis; Ler: Landsberg erecta; PC: Principal component; PCA Principal component analysis; PLS-DA: partial least squares-discriminant analysis

\section{Acknowledgements}

We are grateful to Dr. Giltsu Choi (KAIST, Daejeon, Korea) for providing Arabidopsis mutant seeds. This work was supported by a grant to JRL from the Crop Functional Genomics Center of the 21st Century Frontier Research Program funded by the Korea Ministry of Education, Science and Technology, a grant to JRL from the Marine Extreme Genome Research Center funded by the Korean Ministry of Marine Affairs and Fisheries, and a grant (ABC1001013) to SWK from BioGreen 21 Program funded by the Rural Development Administration.

\section{Author details}

'Biological Resource Center, Korea Research Institute of Bioscience and Biotechnology (KRIBB), 125 Gwahak-ro, Yuseong-gu, Daejeon, 305-806, Korea. 2Plant Systems Engineering Research Center, Korea Research Institute of Bioscience and Biotechnology (KRIBB), 125 Gwahak-ro, Yuseong-gu, Daejeon, 305-806, Korea. ${ }^{3}$ Division of Pharmacognosy, Kyung Hee University, 1 Hoegi dong Dongdaemun-Gu, Seoul, 130-701, Korea.

\section{Authors' contributions}

$J R L$ conceived and directed this study, and drafted the manuscript. SWK and JHK performed most experimental works and wrote the paper with input from co-authors. HJK and YPJ carried out the DART-MS and identified chemical compounds. YKK carried out the statistical analysis. MSA performed Arabidopsis seed management and contributed to the manuscript. All authors read and approved the final manuscript.

\section{Competing interests}

The authors declare that they have no competing interests.

Received: 18 March 2011 Accepted: 10 June 2011

Published: 10 June 2011

\section{References}

1. Gidman E, Goodacre R, Emmett B, Smith AR, Gwynn-Jones D: Investigating plant-plant interference by metabolic fingerprinting. Phytochemistry 2003, 63:705-710.

2. Arbona V, Iglesias DJ, Talon M, Gomez-Cadenas A: Plant phenotype demarcation using nontargeted LC-MS and GC-MS metabolite profiling J Agric Food Chem 2009, 57:7338-7347.

3. Hou CC, Chen CH, Yang NS, Chen YP, Lo CP, Wang SY, Tien YJ, Tsai PW, Shyur LF: Comparative metabolomics approach coupled with cell- and gene-based assays for species classification and anti-inflammatory 
bioactivity validation of Echinacea plants. J Nutr Biochem 2010, 21:1045-1059.

4. Ward JL, Harris C, Lewis J, Beale MH: Assessment of $1 \mathrm{H}$ NMR spectroscopy and multivariate analysis as a technique for metabolite fingerprinting of Arabidopsis thaliana. Phytochemistry 2003, 62:949-957.

5. Kim SW, Ban SH, Jeong SC, Chung HJ, Ko SM, Yoo OJ, Liu JR: Genetic discrimination between Catharanthus roseus cultivars by metabolic fingerprinting using ${ }^{1} \mathrm{H}$ NMR spectra of aromatic compounds. Biotechnol Bioprocess Eng 2007, 12:646-652.

6. Luthria DL, Lin LZ, Robbins RJ, Finley JW, Banuelos GS, Harnly JM: Discriminating between cultivars and treatments of broccoli using mass spectral fingerprinting and analysis of variance-principal component analysis. J Agric Food Chem 2008, 56:9819-9827.

7. Choi HK, Choi YH, Verberne M, Lefeber AW, Erkelens C, Verpoorte R: Metabolic fingerprinting of wild type and transgenic tobacco plants by ${ }^{1} \mathrm{H}$ NMR and multivariate analysis technique. Phytochemistry 2004, 65:857-864.

8. Charlton A, Allnutt T, Holmes $\mathrm{S}$, Chisholm J, Bean S, Ellis N, Mullineaux P, Oehlschlager S: NMR profiling of transgenic peas. Plant Biotechnol J 2004, 2:27-35.

9. Garratt LC, Linforth R, Taylor AJ, Lowe KC, Power JB, Davey MR: Metabolite fingerprinting in transgenic lettuce. Plant Biotechnol I 2005 3:165-174.

10. Mungur R, Glass AD, Goodenow DB, Lightfoot DA: Metabolite fingerprinting in transgenic Nicotiana tabacum altered by the Escherichia coli glutamate dehydrogenase gene. J Biomed Biotechnol 2005, 2005:198-214.

11. Messerli G, Partovi Nia V, Trevisan M, Kolbe A, Schauer N, Geigenberger P, Chen J, Davison AC, Fernie AR, Zeeman SC: Rapid classification of phenotypic mutants of Arabidopsis via metabolite fingerprinting. Plant Physiol 2007, 143:1484-1492.

12. Tian C, Chikayama E, Tsuboi Y, Kuromori T, Shinozaki K, Kikuchi J, Hirayama T: Top-down phenomics of Arabidopsis thaliana: metabolic profiling by one- and two-dimensional nuclear magnetic resonance spectroscopy and transcriptome analysis of albino mutants. J Biol Chem 2007, 282:18532-18541.

13. Ren $Y$, Wang $T$, Peng $Y, X i a B, Q u L$ : Distinguishing transgenic from nontransgenic Arabidopsis plants by ${ }^{1} \mathrm{H}$ NMR-based metabolic fingerprinting. J Genet Genomics 2009, 36:621-628.

14. Belton PS, Colquhoun IJ, Kemsley EK, Delgadillo I, Roma P, Dennis MJ, Sharman M, Holmes E, Nicholson JK, Spraul M: Application of chemometrics to the ${ }^{1} \mathrm{H}$ NMR spectra of apple juices: discrimination between apple varieties. Food Chem 1998, 61:207-213

15. Le Gall G, Colquhoun IJ, Defernez M: Metabolite profiling using ${ }^{1} \mathrm{H}$ NMR spectroscopy for quality assessment of green tea, Camellia sinensis (L.). J Agric Food Chem 2004, 52:692-700.

16. Pongsuwan W, Fukusaki E, Bamba T, Yonetani T, Yamahara T, Kobayashi A: Prediction of Japanese green tea ranking by gas chromatography/mass spectrometry-based hydrophilic metabolite fingerprinting. J Agric Food Chem 2007, 55:231-236.

17. Lee EJ, Shaykhutdinov R, Weljie AM, Vogel HJ, Facchini PJ, Park SU, Kim YK, Yang TJ: Quality assessment of ginseng by ${ }^{1} \mathrm{H}$ NMR metabolite fingerprinting and profiling analysis. J Agric Food Chem 2009, 57:7513-7522.

18. Tianniam S, Bamba T, Fukusaki E: Non-targeted metabolite fingerprinting of oriental folk medicine Angelica acutiloba roots by ultra performance liquid chromatography time-of-flight mass spectrometry. J Sep Sci 2009, 32:2233-2244

19. Fiehn O, Kopka J, Dormann P, Altmann T, Trethewey RN, Willmitzer L: Metabolite profiling for plant functional genomics. Nat Biotechnol 2000, 18:1157-1161.

20. Sumner LW, Mendes P, Dixon RA: Plant metabolomics: large-scale phytochemistry in the functional genomics era. Phytochemistry 2003, 62:817-836.

21. Roessner U, Willmitzer L, Fernie AR: Metabolic profiling and biochemical phenotyping of plant systems. Plant Cell Rep 2002, 21:189-196.

22. Krishnan $P$, Kruger NJ, Ratcliffe RG: Metabolite fingerprinting and profiling in plants using NMR. J Exp Bot 2005, 56:255-265.

23. Hirai MY, Yano M, Goodenowe DB, Kanaya S, Kimura T, Awazuhara M, Arita M, Fujiwara T, Saito K: Integration of transcriptomics and metabolomics for understanding of global responses to nutritional stresses in Arabidopsis thaliana. Proc Natl Acad Sci USA 2004, 101:10205-11210

24. Hirai MY, Klein M, Fujikawa Y, Yano M, Goodenowe DB, Yamazaki Y, Kanaya S, Nakamura Y, Kitayama M, Suzuki H, Sakurai N, Shibata D, Tokuhisa J, Reichelt M, Gershenzon J, Papenbrock J, Saito K: Elucidation of gene-to-gene and metabolite-to-gene networks in arabidopsis by integration of metabolomics and transcriptomics. J Biol Chem 2005, 280:25590-25555.

25. Huang J, Bhinu VS, Li X, Dallal Bashi Z, Zhou R, Hannoufa A: Pleiotropic changes in Arabidopsis f5h and sct mutants revealed by large-scale gene expression and metabolite analysis. Planta 2009, 230:1057-1069.

26. Luo ZB, Janz D, Jiang X, Gobel C, Wildhagen H, Tan Y, Rennenberg H, Feussner I, Polle A: Upgrading root physiology for stress tolerance by ectomycorrhizas: insights from metabolite and transcriptional profiling into reprogramming for stress anticipation. Plant Physiol 2009, 151:1902-1917

27. Dunn WB, Bailey NJ, Johnson HE: Measuring the metabolome: current analytical technologies. Analyst 2005, 130:606-625.

28. Cody RB, Laramee JA, Durst HD: Versatile new ion source for the analysis of materials in open air under ambient conditions. Anal Chem 2005, 77:2297-2302.

29. Banerjee S, Madhusudanan KP, Chattopadhyay SK, Rahman LU, Khanuja SP: Expression of tropane alkaloids in the hairy root culture of Atropa acuminata substantiated by DART mass spectrometric technique. Biomed Chromatogr 2008, 22:830-834.

30. Madhusudanan KP, Banerjee S, Khanuja SP, Chattopadhyay SK: Analysis of hairy root culture of Rauvolfia serpentina using direct analysis in real time mass spectrometric technique. Biomed Chromatogr 2008, 22:596-600.

31. Fernandez FM, Cody RB, Green MD, Hampton CY, McGready R, Sengaloundeth S, White NJ, Newton PN: Characterization of solid counterfeit drug samples by desorption electrospray ionization and direct-analysis-in-real-time coupled to time-of-flight mass spectrometry. ChemMedChem 2006, 1:702-705.

32. Petucci C, Diffendal J, Kaufman D, Mekonnen B, Terefenko G, Musselman B: Direct analysis in real time for reaction monitoring in drug discovery. Anal Chem 2007, 79:5064-5070.

33. Banerjee S, Madhusudanan KP, Khanuja SP, Chattopadhyay SK: Analysis of cell cultures of Taxus wallichiana using direct analysis in real-time mass spectrometric technique. Biomed Chromatogr 2008, 22:250-253.

34. Kim HJ, Jang YP: Direct analysis of curcumin in turmeric by DART-MS. Phytochem Anal 2009, 20:372-377.

35. Morlock $G$, Ueda $Y$ : New coupling of planar chromatography with direct analysis in real time amss spectrometry. J Chromatogr A 2007, 1143:243-251.

36. Vaclavik L, Cjka T, Hrbek V: Ambient mass spectrometry employing direct analysis in real time (DART) ion source for olive oil quality and authenticity assessment. Analytica Chimica Acta 2009, 645:56-63.

37. Koornneef $\mathrm{M}$, Hanhart $\mathrm{CJ}$, van der Veen $\mathrm{JH}$ : A genetic and physiological analysis of late flowering mutants in Arabidopsis thaliana. Mol Gen Genet 1991, 229:57-66

38. Baudry $A$, Caboche $M$, Lepiniec $L: T \pi$ controls its own expression in a feedback regulation involving TTG1 and homologous MYB and bHLH factors, allowing a strong and cell-specific accumulation of flavonoids in Arabidopsis thaliana. Plant J 2006, 46:768-779.

39. Nesi N, Debeaujon I, Jond C, Pelletier G, Caboche M, Lepiniec L: The Tr8 gene encodes a basic helix-loop-helix domain protein required for expression of DFR and BAN genes in Arabidopsis siliques. Plant Cell 2000, 12:1863-1878

40. Nesi N, Jond C, Debeaujon I, Caboche M, Lepiniec L: The Arabidopsis $T 2$ gene encodes an R2R3 MYB domain protein that acts as a key determinant for proanthocyanidin accumulation in developing seed. Plant Cell 2001, 13:2099-2114

41. Reed JW, Nagpal P, Poole DS, Furuya M, Chory J: Mutations in the gene for the red/far-red light receptor phytochrome B alter cell elongation and physiological responses throughout Arabidopsis development. Plant Cell 1993, 5:147-157.

42. Borevitz JO, Xia Y, Blount J, Dixon RA, Lamb C: Activation tagging identifies a conserved MYB regulator of phenylpropanoid biosynthesis. Plant Cell 2000, 12:2383-2394.

43. Lee Y, Yoon HR, Paik YS, Liu JR, Chung WI, Choi G: Reciprocal regulation of Arabidopsis UGT78D2 and BANYULS is critical for regulation of the 
metabolic flux of anthocyanidins to condensed tannins in developing seed coats. J Plant Biol 2005, 48:356-370.

44. Xie DY, Sharma SB, Paiva NL, Ferreira D, Dixon RA: Role of anthocyanidin reductase, encoded by BANYULS in plant flavonoid biosynthesis. Science 2003, 299:396-399

45. Albert $\mathrm{S}$, Delseny M, Devic M: BANYULS, a novel negative regulator of flavonoid biosynthesis in the Arabidopsis seed coat. Plant J 1997, 11:289-299.

46. Devic M, Guilleminot J, Debeaujon I, Bechtold N, Bensaude E, Koornneef M, Pelletier G, Delseny M: The BANYULS gene encodes a DFR-like protein and is a marker of early seed coat development. Plant J 1999, 19:387-398.

47. Debeaujon I, Peeters AJ, Leon-Kloosterziel KM, Koornneef M: The TRANSPARENT TESTA12 gene of Arabidopsis encodes a multidrug secondary transporter-like protein required for flavonoid sequestration in vacuoles of the seed coat endothelium. Plant Cell 2001, 13:853-871.

48. Baudry A, Heim MA, Dubreucq B, Caboche M, Weisshaar B, Lepiniec L: TT2, $\Pi \pi 8$, and TTG1 synergistically specify the expression of BANYULS and proanthocyanidin biosynthesis in Arabidopsis thaliana. Plant J 2004, 39:366-380.

49. Cody RB, Laramee JA, Durst HD: Versatile new ion source for the analysis of materials in open air under ambient conditions. Anal Chem 2005, 77:2297-2302

50. Rummel JL, McKenna AM, Marshall AG, Eyler JR, Powell DH: The coupling of direct analysis in real time ionization to fourier transform ion cyclotron resonance mass spectrometry for ultrahigh-resolution mass analysis. Rapid Comm Mass Spec 2010, 24:784-790.

51. Alonso JM, Stepanova AN, Leisse TJ, Kim CJ, Chen H, Shinn P, Stevenson DK, Zimmerman J, Barajas P, Cheuk R, Gadrinab C, Heller C, Jeske A, Koesema E, Meyers CC, Parker H, Prednis L, Ansari Y, Choy N, Deen H, Geralt M, Hazari N, Hom E, Karnes M, Mulholland C, Ndubaku R, Schmidt I, Guzman P, AguilarHenonin L, Schmid M, Weigel D, Carter DE, Marchand T, Risseeuw E, Brogden D, Zeko A, Crosby WL, Berry CC, Ecker JR: Genome-wide insertional mutagenesis of Arabidopsis thaliana. Science 2003, 301:653-657.

52. Eriksson L, Johansson E, Kettaneh-Wold N, Trygg J, Wikstrom C, Wold S: Multi- and megavariate data analysis part I: Basic principles and applications. Umetrics Academy, Sweden , 22006.

doi:10.1186/1746-4811-7-14

Cite this article as: Kim et al:: A rapid, simple method for the genetic discrimination of intact Arabidopsis thaliana mutant seeds using metabolic profiling by direct analysis in real-time mass spectrometry. Plant Methods 2011 7:14.

\section{Submit your next manuscript to BioMed Central and take full advantage of:}

- Convenient online submission

- Thorough peer review

- No space constraints or color figure charges

- Immediate publication on acceptance

- Inclusion in PubMed, CAS, Scopus and Google Scholar

- Research which is freely available for redistribution

Submit your manuscript at www.biomedcentral.com/submit
Biomed Central 